\title{
Application of Adaptive Noise Cancellation in Transabdominal Fetal Heart Rate Detection Using Photoplethysmography
}

\author{
Kok Beng Gan ${ }^{1}$, Edmond Zahedi² and Mohd. Alauddin Mohd. Ali ${ }^{1}$ \\ 1 Institute of Space Science, National University of Malaysia \\ ${ }^{2}$ School of Electrical Engineering, Sharif University of Technology \\ ${ }^{1}$ Malaysia \\ 2Iran
}

\section{Introduction}

Monitoring the fetal heart rate (FHR) throughout pregnancy empowers the clinician to diagnose fetal well being, characterize fetal development and detect abnormality (Freeman et al. 2003). A non-invasive and low cost system would enable monitoring of normal pregnancies and promote large population studies of fetal physiological development (Freeman et al. 2003). FHR monitoring is an ongoing observation of human fetal physiology. The expected outcome of this early detection is a reduced risk of fetal morbidity and mortality (Philip et al. 2002).

Currently, Doppler ultrasound has been extensively used for FHR detection and obstetric purposes (Hershkovitz et al. 2002), where the standard pre-delivery test of fetal health is the fetal non-stress test (NST). These tests are routinely performed at the hospital where continuous-wave instruments are more popular than pulsed ones. The use of Doppler ultrasound in the first trimester is generally not recommended as a routine (Hershkovitz et al. 2002) as it may increases the occurrence of intrauterine growth restriction. Besides that, the FHR measurements using Doppler ultrasound are not always reliable (Karlsson et al. 2000) due to the complexity of the Doppler signal and the effects of fetal and maternal breathing. An alternative to ultrasound is using the fetal electrocardiogram (FECG). In direct (invasive) fetal electrocardiogram (FECG), the FHR could be obtained by attaching scalp electrode to the fetal scalp after the rupture of the membrane (Khandpur 2004). During invasive FECG recording the uterus may be perforated leading to its infection, besides possible scalp injuries to the fetus (Khandpur 2004). The other approach is non-invasive FECG but FECG signals have a low (signal to noise ratio) SNR due to the interference from noise, maternal electrocardiogram (MECG) and electromyogram (EMG). The application of non-invasive FECG requires multiple leads and advanced digital signal processing techniques (Najafabadi et al. 2006). It is worth mentioning that commercial devices operating on non-invasive FECG are not available at this moment.

Optical techniques has received a considerable attention in biomedical diagnostic and monitoring of biological tissues such as brain imaging, breast imaging and for fetal heart rate detection and oxygen saturation measurement due to its theoretical advantages in 
comparison with other modalities. Continuous wave near infrared (NIR) spectroscopy has been applied to trans-abdominal fetal pulse oximetry (Ramanujam et al. 2000; Chance 2005; Zourabian et al. 2000; Nioka et al. 2005; Vintzileos et al. 2005). The system consists of NIR sources (halogen lamps) and a photomultiplier as detection unit (Ramanujam et al. 2000; Chance 2005). The generated heat was justified by using cooling fans for the halogen lamps. Recently, trans-abdominal oxygen saturation $\left(\mathrm{S}_{\mathrm{p}} \mathrm{O}_{2}\right)$ in animal (Nioka et al. 2005) and human fetuses were successfully obtained in the laboratory (Vintzileos et al. 2005). However, the proposed techniques require high power (a total of $80 \mathrm{~W}$ optical power) and a relatively expensive detection unit (photo-multiplier).

In this project, we propose to design and develop a low-power optical FHR monitor. The signal of interest is the photoplethysmogram (PPG), which is generated when a beam of light is modulated by blood pulsations. PPG is a noninvasive technique for detecting blood volume changes in living tissue by optical means consisting of a light emitting diode (LED) and a photo-detector. One of the potential applications of the PPG technology is noninvasive fetal heart rate detection through the maternal abdomen. In this application, the light intensity is modulated by the mother as well as fetal blood circulation, producing a combined signal which needs to be separated via digital signal processing (DSP) techniques. The design of a fixed filter would not be adequate as the frequency spectrum of the noise (maternal PPG) overlaps with the desired signal (fetal PPG). The adaptive filter will automatically adjust its coefficients therefore achieve the high degree of noise rejection.

Such an approach - based on adaptive noise cancellation (ANC) - has been evaluated for extraction of the fetal heart-rate using PPG signals from the maternal abdomen. A simple optical model has been proposed in which the maternal and fetal blood pulsations result in emulated signals where the lower SNR limit (fetal to maternal) is $-25 \mathrm{~dB}$ (Zahedi \& Beng, 2008). It is shown that the RLS algorithm is capable to extract the peaks of the fetal PPG from these signals, corresponding to typical values of maternal and fetal tissues.

Subsequently, an optical fetal heart rate detection (OFHR) system has been designed and developed using low-cost, low-power IR light $(890 \mathrm{~nm}$ with optical power $<68 \mathrm{~mW})$ and a commercially available silicon photo-detector (Gan et al. 2009). Previous literature (Ramanujam et al. 2000; Chance 2005; Zourabian et al. 2000; Nioka et al. 2005; Vintzileos et al. 2005; Choe et al. 2003) shows that the Source-Detector separations depends on the type of sources and the photo-detectors implemented in their studies. Since in our work the developed instrument utilizes low optical power, the source-to-detector separation plays an important role as it affects the detectivity of the photo-detector. This chapter discusses the selection of S-D separation for the OFHR system based on the ANC limit and photodetector's noise. The implementation of the ANC algorithm in OFHR system is also discussed and the clinical trial results are also reported.

\section{Materials and methods}

\subsection{Adaptive noise cancellation}

Conventional digital signal processing techniques do exist to extract a desired biomedical signal from a mixed signal which is usually contaminated by unwanted noises. Adaptive filters are used for non-stationary signals where a sample-by-sample adaptation process is required (Vaseghi, 2000; Widrow et al., 1975). Applications of adaptive filtering include multi-channel noise reduction, radar or sonar signal processing, channel equalization for cellular mobile phones, echo cancellation and low delay speech coding. This section 
discussed the concept of the adaptive filtering, adaptive algorithm and the Recursive Least Square (RLS) algorithm.

\subsubsection{Concept of adaptive filtering}

Adaptive filters consist of two distinct parts: a digital filter and the corresponding adaptive algorithm, used to adjust the coefficients of the filter (Figure 1). In these algorithms, the error signal $e(n)$ defined as the difference between the output of the filter $(y(n))$ and a primary input signal $(d(n))$, is minimized according to a least squares error criterion (Ifeachor \& Jervis, 2002).

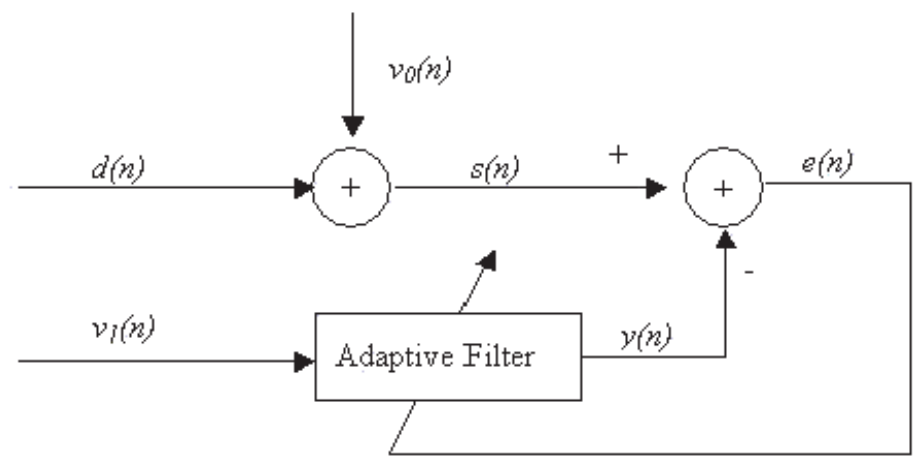

Fig. 1. ANC system

The desired signal $d(n)$ (Figure 1) is contaminated by an uncorrelated noise signal $v_{0}(n)$, where $n$ is the running time index. The result $d(n)+v_{0}(n)$ is the primary measurement signal $s(n)$. The reference input, $v_{1}(n)$ is only correlated with $v_{0}(n)$ and fed to an adaptive FIR filter. The output of the FIR adaptive filter $y(n)$ is subtracted from the primary input $s(n)$ to produce the error signal $e(n)$ :

$$
e(n)=d(n)+v_{0}(n)-y(n)
$$

The adaptive filter uses $e(n)$ to adjust its own impulse response to produce an output $y(n)$ as close a replica as possible to $v_{0}(n)$. Squaring and applying the expectation operator to both sides of Equation 1:

$$
E\left\{e^{2}(n)\right\}=E\left\{d^{2}(n)\right\}+E\left\{\left(v_{0}(n)-y(n)\right)^{2}\right\}-2 E\left\{d(n)\left(v_{0}(n)-y(n)\right)\right\}
$$

$d(n)$ being uncorrelated with $v_{0}(\mathrm{n})$ and $v_{1}(\mathrm{n}), E\left\{d(n)\left(v_{0}(n)-y(n)\right)\right\}=0$. Therefore Equation 2 can be simplified:

$$
E\left\{e^{2}(n)\right\}=E\left\{d^{2}(n)\right\}+E\left\{\left(v_{0}(n)-y(n)\right)^{2}\right\}
$$

An iterative procedure minimizes $E\left\{e^{2}(n)\right\}$, which will occur when $y(n)=v_{0}(n)$ (ideal situation) producing $e(n)=d(n)$. 


\subsubsection{Adaptive algorithm}

In most adaptive systems, the digital filter in Figure 2 is realized using a transversal or finite impulse response (FIR) structure. The FIR structure is the most widely used because of its simplicity and stability.

A $m$ th-order adaptive transversal filter is a linear time varying discrete-time system that can be represented by:

$$
y(n)=\sum_{i=0}^{m-1} w_{i}(n) v_{1}(n-i)
$$

where $w_{i}(n)$ is the adjustable weight and $v_{1}(n)$ and $y(n)$ are the input and output of the filter. The filter output is a time varying linear combination of the past input (Figure 2).

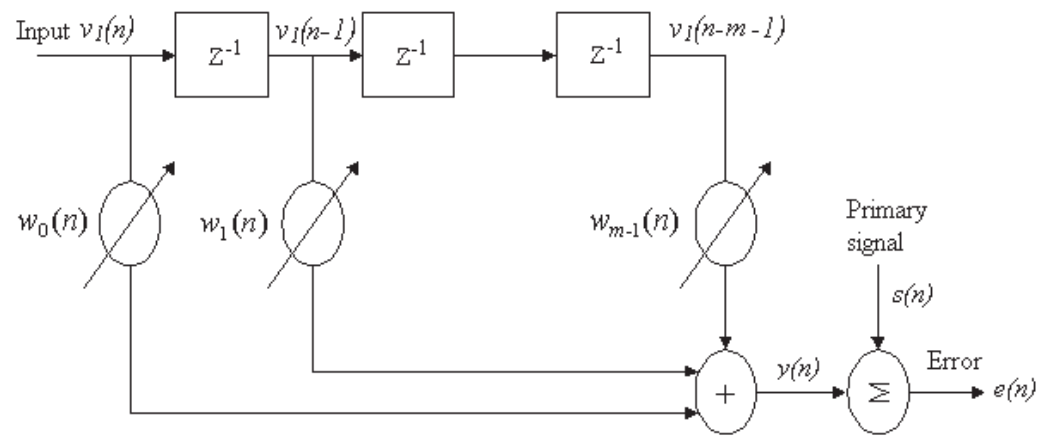

Fig. 2. Illustration of the configuration of an adaptive filter

Adaptive algorithm are used to adjust the coefficient of the digital filter (Figure 2) such that the error signal $e(n)$, is minimized according to the mean square error and least squares error criterion (Ifeachor \& Jervis, 2002). Common adaptation algorithms are least mean square (LMS) and the RLS. The RLS algorithm minimizes the sum of the square of the error whereas the LMS algorithm minimizes the mean square error. In terms of the computational and storage requirements, the LMS algorithm is the most efficient and does not suffer from the numerical instability problem (Ifeachor \& Jervis, 2002). However, the recursive least square (RLS) algorithm has superior convergence properties (Ifeachor \& Jervis, 2002). It is suitable for offline processing where computational requirement is not an issue.

\subsubsection{Linear least-square error estimation}

The principle of least-squares (LS) was introduced by the German mathematician Carl Friedrich Gauss, who used it to determine the orbit of the asteroid ceres in 1821 by formulating the estimation problem as an optimization problem (Manolakis et al., 2005).

The least-square approach provides a mechanism for designing fixed filters when the properties of the signal source are known. More importantly, it provides a vehicle for adaptive filter design that can operate in an environment of changing signal properties. The source signal is modeled as the output of a linear discrete-time system with parameters which are either known for the fixed algorithm or unknown in the adaptive case. Noise added to the observations completes the signal description. The least-square algorithm is then required to 
do the "best" filtering of the signal, employing as much of the priori signal and noise models as is known. If these priori properties are unknown, then the LS algorithm is required to identify the changed conditions and to adapt its parameters to the new signal environment.

The basic idea of the LS method is shown in Figure 3. An output signal, $s(n)$ measured at the discrete time, $n$ in response to a set of input signal, $v_{1}(n)$. The input and output signals are related by the simple regression model.

$$
s(n)=\sum_{i=0}^{m-1} w_{i}(n) v_{1}(n)+e(n)
$$

where $e(n)$ is the measurement errors and $w_{i}(n)$ is the adjustable weight with $m$ th order.

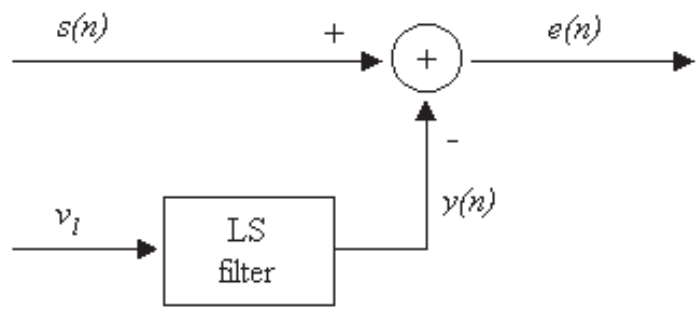

Fig. 3. An illustration of the basic idea of the LS method

The estimation error is defined as

$$
\begin{gathered}
e(n)=s(n)-\sum_{i=0}^{m-1} w_{i}(n) v_{1}(n) \\
=s(n)-\boldsymbol{w}^{\boldsymbol{T}} \boldsymbol{v}_{1}
\end{gathered}
$$

where $v_{1}=\left[v_{1}(n), v_{1}(n-1), \ldots, v_{1}(n-m-1)\right]^{T}$ and $w=\left[w_{0}(n), w_{1}(n), \ldots, w_{m-1}(n)\right]^{T}$. The filter weight, $w_{i}(n)$ are determined by minimizing the sum of the squared errors

$$
E \Delta \sum_{n=0}^{n-1}|e(n)|^{2}
$$

that is, the energy of the signal.

To explore the relation between the filter coefficient, $w$, and the error signal, $e(n)$, Equation 6 can be written in matrix form for $N$ samples measurement of the signals $[s(0), s(1), \ldots, s(N-1)]$ and signals $\left[v_{1}(n), v_{1}(1), \ldots, v_{1}(N-1)\right]$ as

$$
\left(\begin{array}{c}
e(0) \\
e(1) \\
e(2) \\
\vdots \\
e(N-1)
\end{array}\right)=\left(\begin{array}{c}
s(0) \\
s(1) \\
s(2) \\
\vdots \\
s(N-1)
\end{array}\right)-\left(\begin{array}{ccccc}
v_{10}(0) & v_{11}(0) & v_{12}(0) & \ldots & v_{1 m-1}(0) \\
v_{10}(1) & v_{11}(1) & v_{12}(1) & \ldots & v_{1 m-1}(1) \\
v_{10}(2) & v_{11}(2) & v_{12}(2) & \ldots & v_{1 m-1}(2) \\
\vdots & \vdots & \vdots & \ddots & \vdots \\
v_{10}(N-1) & v_{11}(N-1) & v_{12}(N-1) & \ldots & v_{1 m-1}(N-1)
\end{array}\right)\left(\begin{array}{c}
w_{0} \\
w_{1} \\
w_{2} \\
\vdots \\
w_{m-1}
\end{array}\right)
$$


or more compactly as

$$
e=s-V w
$$

where

$$
\begin{aligned}
& \boldsymbol{e} \triangleq[e(0), e(1), \ldots, e(N-1)]^{T} \quad \text { error data vector }(N \times 1) \\
& s \underline{\Delta}[s(0), s(1), \ldots, s(N-1)]^{T} \quad \text { primary data vector }(N \times 1) \\
& V \underline{\underline{\Delta}}\left[\boldsymbol{v}_{1}(0), \boldsymbol{v}_{1}(1), \ldots, \boldsymbol{v}_{1}(N-1)\right]^{T} \quad \text { input data matrix }(N \times m) \\
& w \triangleq\left[w_{0}, w_{1}, \ldots, w_{m-1}\right]^{T} \quad \text { weight vector }(m \times 1)
\end{aligned}
$$

where $v_{1}(n) \underline{\Delta}\left[v_{10}(0), v_{11}(1), \ldots, v_{1 m-1}(n)\right]$. The energy of the error vector, that is the sum of squared elements of the squared error vector, is given by the inner vector product as:

$$
\begin{gathered}
e^{T} e=(s-V w)^{T}(s-V w) \\
=s^{T} s-s^{T} V w-V^{T} w^{T} s+V^{T} w^{T} V w
\end{gathered}
$$

The gradient of the squared error function with respect to the filter coefficients is obtained by differentiating Equation 11 with respect to $w$ as:

$$
\frac{\partial e^{T} e}{\partial w}=-2 s^{T} V+2 w^{T} V^{T} V
$$

The filter coefficients are obtained by setting the gradient of the squared error function of Equation 12 to zero and yield:

$$
\left(V^{T} V\right) w=V^{T} s
$$

or

$$
w=\left(V^{T} V\right)^{-1} V^{T} S
$$

Note that the matrix $V^{T} \boldsymbol{V}$ is a time-averaged estimate of the autocorrelation matrix of the input signal, $\boldsymbol{R}_{y y}$ and the vector $\boldsymbol{V}^{T} \boldsymbol{S}$ is a time-averaged estimate of the cross-correlation vector of the input and the primary signals, $\boldsymbol{r}_{y x}$

\subsubsection{Recursive least square algorithm}

The RLS algorithm is based on the least-square method (Ifeachor \& Jervis, 2002; Haykin, 2002). In recursive implementations of the method of least squares, the computation is started with known initial conditions and use the information contained in new data samples to update the old estimates. The RLS adaptive filters are designed so that the updating of the coefficients is always achieved the minimization of the sum of the squared errors. The RLS adaptive algorithm for updating the coefficients of the FIR filter is superior to the LMS algorithm in convergence properties, eigen value sensitivity, and excess MSE. The price paid for this improvement is additional computational complexity.

The computation of $w$ in Equation 14 requires time-consuming computation of the inverse matrix. With the RLS algorithm the estimate of $w$ can be updated for each new set of data 
acquired without repeatedly solving the time-consuming matrix inversion directly. A suitable RLS algorithm can be obtained by exponential weighting the data to remove gradually the effect of old data on $w$ and to allow the tracking of slowly varying signal characteristic.

The derivation of the RLS algorithm can be found in the report (Gan, 2009) and the RLS algorithm can be summarized as follows:

Input signals: $v_{1}(n)$ and $d(n)$

Initial values:

$$
\begin{gathered}
\boldsymbol{\Phi}_{y y}(n)=\delta^{-1} \boldsymbol{I} \\
\boldsymbol{w}(0)=\boldsymbol{w}_{I}
\end{gathered}
$$

For $n=1,2, \ldots$, compute

1. Filter gain vector update :

$$
\boldsymbol{k}(n)=\frac{\lambda_{f}^{-1} \boldsymbol{\Phi}_{y y}(n-1) \boldsymbol{v}_{\boldsymbol{I}}(n)}{1+\lambda_{f}^{-1} \boldsymbol{v}^{T}(n) \boldsymbol{\Phi}_{y y}(n-1) \boldsymbol{v}_{\boldsymbol{I}}(n)}
$$

2. Error signal equation:

$$
e(n)=d(n)-\boldsymbol{w}^{T}(n-1) \boldsymbol{v}_{1}(n)
$$

3. Filter coefficients adaptation:

$$
\boldsymbol{w}(n)=\boldsymbol{w}(n-1)-\boldsymbol{k}(n) e(n)
$$

4. Inverse correlation matrix update:

$$
\boldsymbol{\Phi}_{y y}(n)=\lambda_{f}^{-1} \boldsymbol{\Phi}_{y y}(n-1)-\lambda_{f}^{-1} \boldsymbol{k}(n) \boldsymbol{v}_{\boldsymbol{I}}^{\boldsymbol{T}}(n) \boldsymbol{\Phi}_{y y}(n-1)
$$

\subsection{Photoplethysmography}

Photoplethysmograph is an optoelectronic method for measuring and recording changes in the volume of body parts such as finger and ear lobes caused by the changes in volume of the arterial oxygenated blood, associated with cardiac contraction (Bronzino 2000). A sample of few normal periodic PPG pulse waves is shown in Figure 4, where the steep rise and dicrotic notch on the falling slope are clearly visible. When light travels through a biological tissue (earlobe or finger), it is absorbed by different absorbing substances. Primary absorbers are the skin pigmentation, bones and the arterial and venous blood. The characteristics of the PPG pulse are influenced by arterial ageing and arterial disease (Allen \& Murray 2000).

The emitted light either red or infrared light emitting diode is detected by a photodetector. The time varying signals of the detected signal is called PPG. The PPG signal contains AC and DC components: the AC component is mainly due to the arterial blood pulsation and the DC component comes from the non-pulsating arterial blood, venous blood and other tissues. 


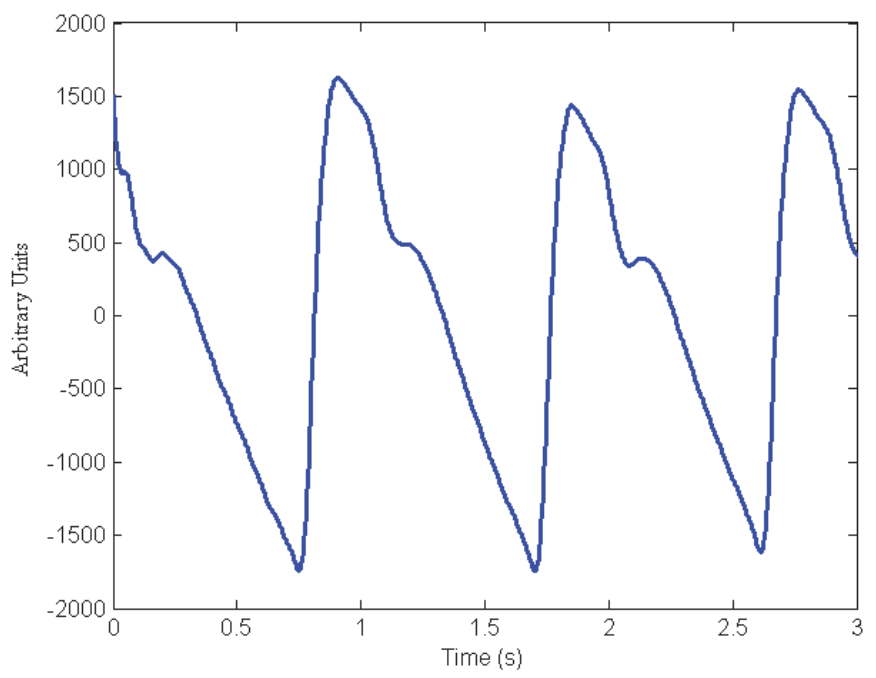

Fig. 4. Typical PPG pulse wave signal acquired in our laboratory

The probes can be of two types, transmission or reflection. A transmission probe measures the amount of light that passes through the tissue as in a finger clip probe. The photodiode is located on the opposite side of the LED and the tissue is located between them. A reflectance probe measures the amount of light reflected to the probe. However, the detected light intensity of a reflectance probe is weaker than the transmission probe with the same source to detector separation.

In this application, transmission probes are not suitable due to the very long optical path that the light would have to travel to the photo-detector which is located opposite sides of the maternal abdomen (Zahedi \& Beng, 2008). The reflectance probe becomes the method of choice where the photo-detector is placed on the same body surface (abdomen) making the measurement of abdominal PPG signal possible (Zahedi \& Beng, 2008).

\subsection{Photo-detector noise}

When designing an optical instrument, the photo-detector is an essential component. Selection of an appropriate photo-detector resulted in better signal quality of the acquired signals. The noise floor of the photo-detector will determine the maximum S-D separation which is useful in the optical instruments.

Currently, the low noise photo-detector (from Edmund Optics Inc.) with noise equivalent power as low as $1.8 \times 10^{-14} \mathrm{~W} / \mathrm{Hz}^{1 / 2}\left(0.051 \mathrm{~cm}^{2}\right)$ (W57-522, Edmund Optics, Inc.) and $8.6 \times 10^{-14}$ $\mathrm{W} / \mathrm{Hz}^{1 / 2}\left(1.00 \mathrm{~cm}^{2}\right)$ (W57-513, Edmund Optics, Inc.). Noise equivalent power is the incident optical power required to produce a signal on the photo-detector that is equal to the noise when the SNR is equal to one. These silicon photo-detectors are then utilized in the following analysis. 


\begin{tabular}{ccccc}
\hline $\begin{array}{c}\text { Photo-detector area } \\
\left(\mathrm{cm}^{2}\right)\end{array}$ & $\begin{array}{c}R \\
(\mathrm{~A} / \mathrm{W})\end{array}$ & $\begin{array}{c}R_{\text {sh }} \min \\
(\mathrm{M} \Omega)\end{array}$ & $\begin{array}{c}\text { Bandwidth } \\
(\mathrm{Hz})\end{array}$ & $\begin{array}{c}I_{P N} \\
(\mathrm{~A})\end{array}$ \\
\hline & & & 100 & $8.29 \times 10-14$ \\
0.051 & 0.62 & 600 & 1000 & $2.63 \times 10-13$ \\
& & & 10000 & $8.29 \times 10-13$ \\
& & & 100000 & $2.63 \times 10-12$ \\
& & & 100 & $3.71 \times 10-13$ \\
& 0.62 & 30 & 1000 & $1.17 \times 10-12$ \\
& & & 10000 & $3.71 \times 10-12$ \\
& & & 100000 & $\times 10-11$ \\
\hline
\end{tabular}

Table 1. $P_{\text {Noise }}$ during photovoltaic operation at various bandwidths

The photo-detector can either operate in photovoltaic or photo-conductance condition. Photovoltaic operation offered a low noise system compared to the photo-conductance operation. Shot noise (due to the dark current) is the dominant noise component during photo-conductance operation. Small photo-detector's active area resulted in lower noise level compared to the large photo-detector's active area. Since strong scattering process for the human tissue dispersed the light in random fashion (Bronzino, 2000), large photodetector's active area increases the probability of detecting photons that exit from the maternal layer. Therefore, photo-detector with $1 \mathrm{~cm}^{2}$ area is proposed for the optical fetal heart rate instrument. This value has thus been used in the rest of this work. Table 1 showed the proposed silicon photo-detector's noise, $P_{\text {Noise }}$ during photovoltaic operation at various bandwidths. It shows that photo-detector's noise increases with its bandwidth.

\section{Results and discussions}

This section discusses the determination of S-D separation based on the limit of ANC operation. Results obtained in previous work (Zahedi \& Beng, 2008) encouraged us to take one step forward via practical implementation of the circuitry whereas digital synchronous detection is utilized to further enhance the SNR. The design and development of the OFHR system is described and results of the clinical trial are also reported.

\subsection{Adaptive noise cancellation and the limit of the photo-detector}

Since the adaptive noise canceling limit is $-34.7 \mathrm{~dB}$, the photo-detector used in the optical fetal heart rate instrument must be able to detect fetal signal at this limit. By using Equation 19 , the expected fetal optical power, $P_{F}$ at $-34.7 \mathrm{~dB}$ is estimated and tabulated in Table 2 .

$$
10 \log _{10}\left[\frac{P_{F}}{P_{M+a m}}\right]=-34.7 d B
$$

where $P_{F}$ is the estimated fetal optical power, $P_{M+a m}$ is the optical power at photo-detector using Monte Carlo simulation and $-34.7 \mathrm{~dB}$ is the limit of the ANC operation. These values were obtained through Monte-Carlo simulation using a three-layered tissue model (maternal, amniotic, and fetal) (Zahedi \& Beng, 2008). Optical properties (scattering and 
absorption coefficients) of the tissue model as well as respective thicknesses were obtained from previous studies (Ramanujam et al. 2000; Gan, 2009), and simulation results were based on the launching of two million photons with $1 \mathrm{~mW}$ optical power. The detailed discussion of the Monte-Carlo simulation can be found in the previous report (Gan, 2009) and will not be further discussed here.

From Figure 5, when S-D separation larger than $4 \mathrm{~cm}(6 \mathrm{~cm}, 8 \mathrm{~cm}$ and $10 \mathrm{~cm})$, the expected optical power is below the photo-detector noise level. At $2 \mathrm{~cm}$ and $4 \mathrm{~cm}$ source to detector separation, the expected fetal optical powers, $2293.99 \times 10^{-12} \mathrm{~W} / \mathrm{cm}^{2}$ and $5.94 \times 10^{-12} \mathrm{~W} / \mathrm{cm}^{2}$ respectively, are higher than the photo-detector's noise $\left(1.17 \times 10^{-12} \mathrm{~W} / \mathrm{cm}^{2}\right)$ level. The photodetector is assumed to be operated at the photovoltaic condition with $1000 \mathrm{~Hz}$ bandwidth and $1 \mathrm{~cm}^{2}$ active area. Therefore, source to detector separation of $4 \mathrm{~cm}$, which results in $70 \%$ of optical power from fetal layer, is suitable to use with this low noise photo-detector. At 890 $\mathrm{nm}$ and $4 \mathrm{~cm}$ source-detector separation, the receiver sensitivity is optimized by considering the limitation of the adaptive filter in FHR detection.

\begin{tabular}{ccc}
\hline $\begin{array}{c}\text { Source to detector separation } \\
(\mathrm{cm})\end{array}$ & $\begin{array}{c}\text { Expected signal level, } \\
P_{M+a m} \\
\left(\times 10^{-9}\right)\end{array}$ & $\begin{array}{c}\text { Expected } P_{F} \text { signal level of } \\
-34.7 \mathrm{~dB} \\
\left(\times 10^{-12}\right)\end{array}$ \\
\hline 2 & 6767.09 & 2293.99 \\
4 & 17.53 & 5.94 \\
6 & 0.31 & 0.11 \\
8 & 0.37 & 0.13 \\
10 & 0.09 & 0.03 \\
\hline
\end{tabular}

Table 2. Expected $P_{F}$ signal level (-34.7 dB) at various source to detector separation

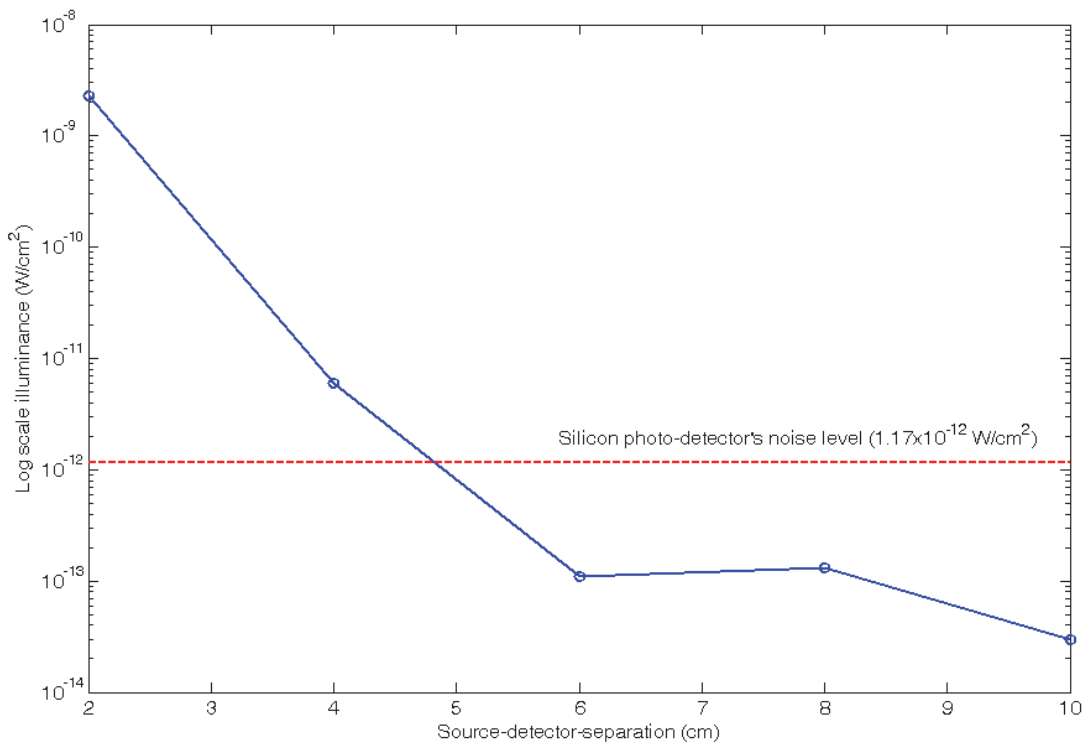

Fig. 5. Estimated $P_{F}(-34.7 \mathrm{~dB})$ at $2.5 \mathrm{~cm}$ fetal depth 


\subsection{Implementation of ANC in transabdominal fetal Heart rate detection using PPG}

In our work (Gan et al. 2009), a low-power optical technique is proposed based on the PPG to non-invasively estimate the FHR. A beam of LED light $(<68 \mathrm{~mW})$ is shone to the maternal abdomen and therefore modulated by the blood circulation of both mother and fetus whereas maximum penetration is achieved at a wavelength of $890 \mathrm{~nm}$. This mixed signal is then processed by an adaptive filter with the maternal index finger PPG as reference input. Figure 6 shows the optical fetal heart rate detection (OFHR) system block diagram whereas the implementation by using National Instrument hardware and LabVIEW software are illustrated in Figure 7 and Figure 8. In the OFHR system, the fetal probe (primary signal) is attached to the maternal abdomen using a Velcro belt to hold the IR-LED and photo-detector, separated by $4 \mathrm{~cm}$. The reference probe is attached to the mother's index finger as generally practiced in pulse oximetry. As the selected IR-LED could only emit a maximum optical power of $68 \mathrm{~mW}$, the OFHR system operates with an optical power less than the limit of $87 \mathrm{~mW}$ specified by the International Commission on Non-Ionizing Radiation Protection (ICNIRP) (International Commission on Non-Ionizing Radiation Protection, 2000). In order to modulate the IR-LED, the modulation signal is generated at a frequency of $725 \mathrm{~Hz}$ using software subroutine through a counter port (NI-USB 9474) to the LED driver (Figure 6).

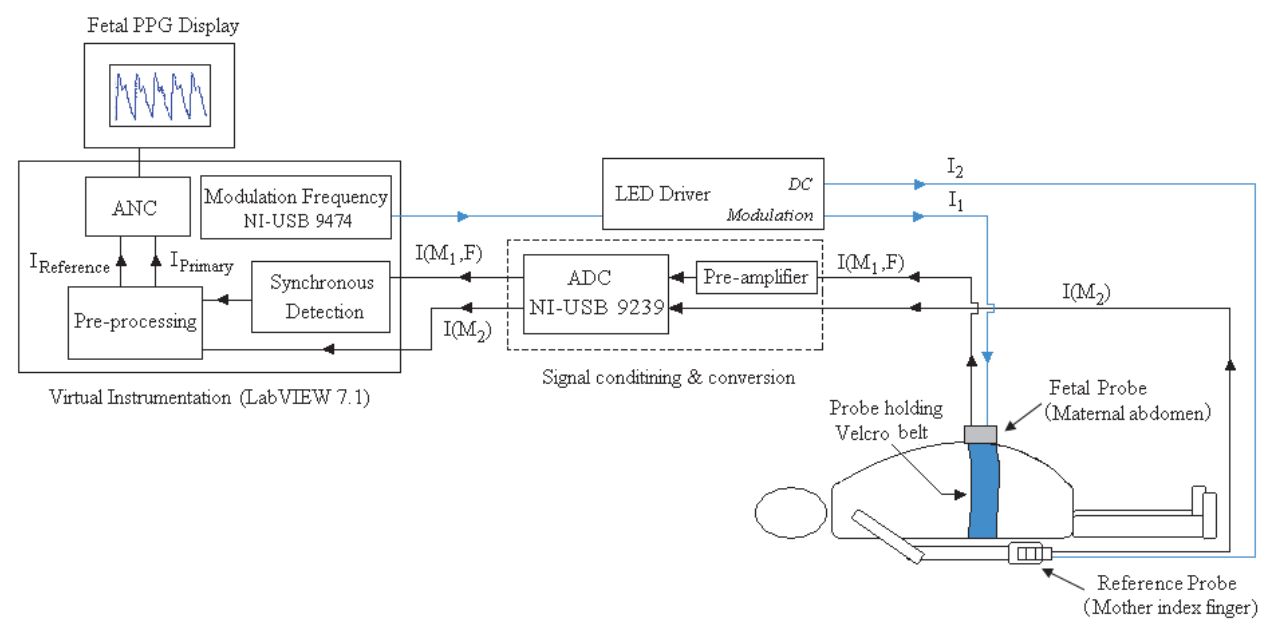

Fig. 6. OFHR system block diagram showing the hardware modules have been implemented in LabVIEW.

The diffused reflected light from the maternal abdomen, detected by the low-noise photodetector, is denoted as $I\left(M_{1}, F\right)$, where $M_{1}$ and $F$ denote the contribution to the signal from the mother abdomen and fetus, respectively. A low-noise $\left(6 \mathrm{nV} / \mathrm{Hz}^{1 / 2}\right)$ transimpedance amplifier is utilized to convert the detected current to a voltage level. The reference probe (mother's index finger) consists of an IR-LED and a solid-state photodiode with an integrated preamplifier. The signal from this probe is denoted as $I\left(M_{2}\right)$, where $M_{2}$ refers to the maternal contribution. Synchronous detection is not required at this channel as the finger photoplethysmogram has a high signal to noise ratio (SNR).

Detected signals from both probes are simultaneously digitized with a 24-bit resolution data acquisition card (NI-USB 9239, National Instruments, Inc.) at a rate of $5.5 \mathrm{kHz}$. The 
demodulation, digital filtering, and signal estimation are all performed in the digital domain. Software implementation consists of generating a modulation signal, a synchronous detection algorithm, down-sampling, high-pass filtering and ANC algorithm (Zahedi \& Beng, 2008). The entire algorithm and part of the instrument have been implemented using Laboratory Virtual Instrumentation Engineering Workbench (LabVIEW 7.1, from National Instruments, Inc.). After pre-processing and applying the ANC algorithm (Figure 9), the fetal signal and the fetal heart rate are displayed. The FHR is found by estimating the prominent peak of the power spectral density using the Yule-Walker autoregressive (AR) method (order of 20).

Figure 7 shows the laboratory prototype and the graphical user interface of the OFHR system (in Figure 7, left) where the maternal index finger PPG (top), the abdominal PPG (middle) and the estimated fetal PPG (bottom) are presented. There are three types of

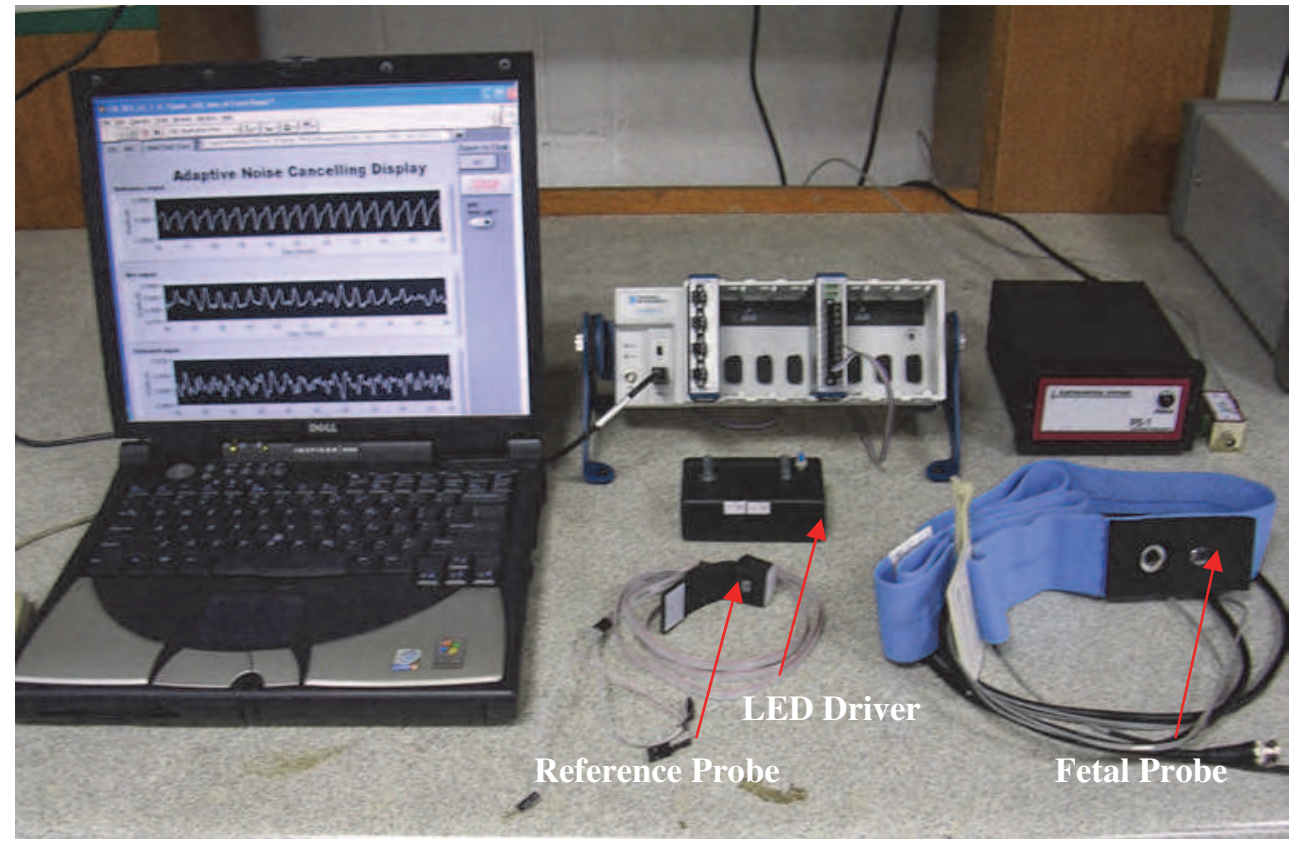

Fig. 7. OFHR prototype

selectable displays (Figure 8) namely digital synchronous or lock-in amplifier (LIA), ANC and heart rate trace. The purpose of the first two displays is to assist development and the third one (Figure 8) indicates FHR values versus time (clinical application). The user can either save the data to the personal computer for further analysis or just display it online.

Finally, a total of 24 data sets were acquired from six subjects at $37 \pm 2$ gestational weeks from the Universiti Kebangsaan Malaysia Medical Centre. This study was reviewed and approved by the University Ethical Committee and written consent was obtained from all patients who participated in this study after the procedure was clearly explained to them. The process for subject recruitment and data acquisition are complied with the rules and regulation as stated in Good Clinical Practice. 


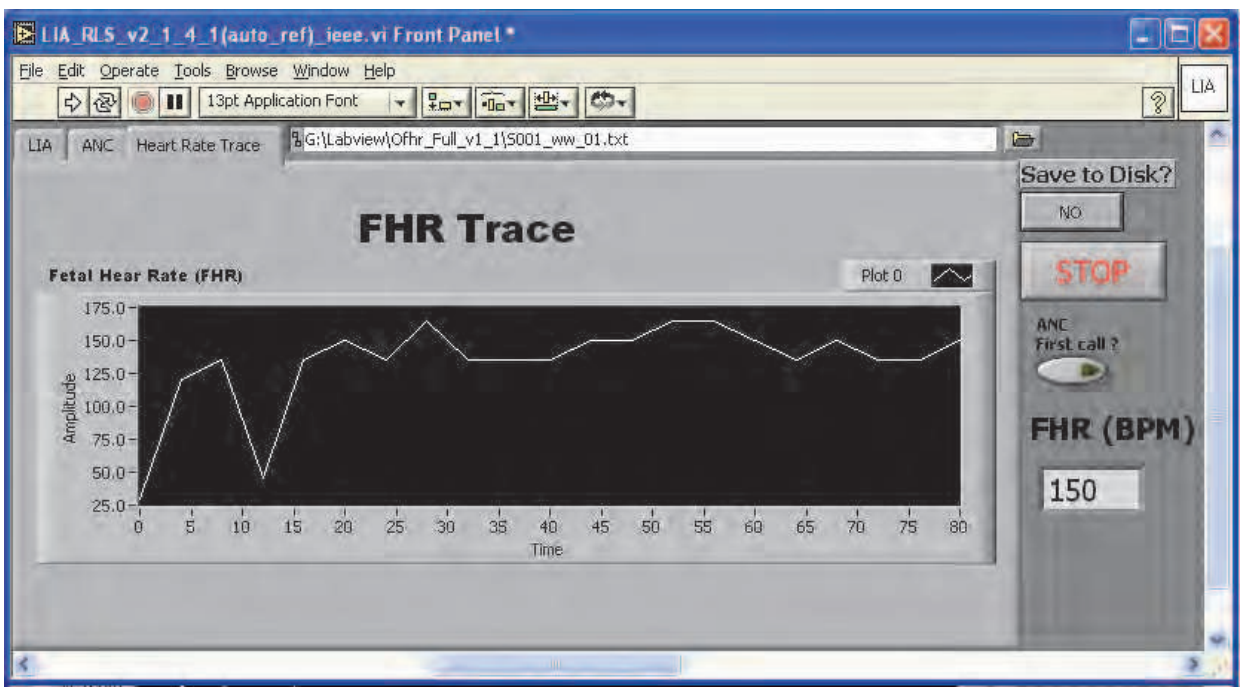

Fig. 8. Graphical User Interface of OFHR system. FHR trace menu

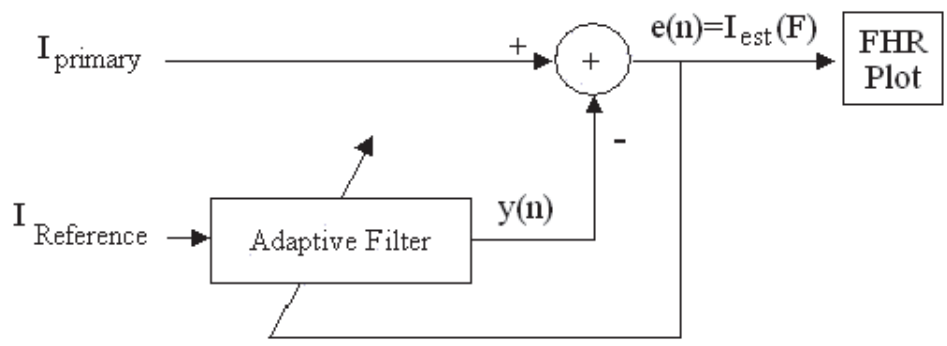

Fig. 9. ANC block diagram

In this study, all fetuses were singleton with gestation weeks from 30 week to 40 week. Subjects with twin pregnancies, anterior placed placenta, obesity (BMI $\geq 30)$, gestational diabetes mellitus (GDM) and hypertension were excluded from this study. In addition, all fetuses in this study were found to be healthy by the obstetrician and born naturally (vaginally) without any complication.

During the data acquisition, the fetal probe is fixed to a maternal abdomen and the reference probe on her index finger in semi-Fowler position. The data analysis shows a correlation coefficient of 0.97 (p-value < 0.001) between optical and ultrasound FHR with a maximum error of $4 \%$. Clinical results indicate that positioning the probe over the nearest fetal tissues (not restricted to head or buttocks) improves signal quality and therefore detection accuracy.

\section{Conclusions}

A low power OFHR detection system has been designed and developed using low cost, very low power $(<68 \mathrm{~mW})$ IR light and a commercially available silicon photo-detector. The digital synchronous detection and adaptive filtering techniques have been successfully 
implemented using LabVIEW 7.1. By applying digital synchronous detection and adaptive filtering techniques the FHR was determined with acceptable accuracy (maximum error of $4 \%$ ) when compared to Doppler ultrasound. Attested by clinical results the probe positioning influences the acquired signal's quality and therefore affects the FHR results. Locating the nearest fetal tissues (not restricted to head or buttocks) to the probe will help to increase the signal quality and FHR determination accuracy.

The limitations of the optical technique are due to the presence of motion artifacts and sensitivity to the probe placement. The presence of motion artifacts may cause loss of correlation between the reference signal and the noise source (maternal PPG) in the mixed signal recorded from the maternal abdomen. The performance of the adaptive filtering scheme will suffer as a consequence, making the probe placement and stability an important criterion. Besides that, finding a proper location is needed in order to get signals with good SNR.

For the future development, by using an array of sensors to automatically select the channel with the highest SNR will eliminate the positioning problem. The topology of the sources and the photo-detector has to be determined. For the cost effective design, it is recommends that more light sources are used instead of photo-detectors. A wearable system will make the device more convenient for clinical applications in the near future. To ensure real-time and low-power function, the whole system can be implemented using embedded processor. The FHR will be wirelessly transmitted to another computing platform (PC or PDA) for further analysis, storage and transmission (to the nursing entity at a clinic). The main performance factors which will be considered are robustness, battery life, weight, dimensions and ergonomy. It is thought that the using of the selected platform (ARM) implementation will lead to a sufficiently low-cost bill-of material for the final product. During development phase, EMC directives will be taken into account so that the system's operation does not affect nor will be affected by other electronic devices. As a by-product of the project and contribution to the scientific community, it is proposed that acquired data during the project to be made available to a public data-base of biological signals (www.physionet.org) maintained by MIT in the USA.

\section{Acknowledgment}

This work has been partially supported by research university grant UKM-AP-TKP-07-2009. The authors would like to express their gratitude to Prof. Dr. M. A. J. M. Yassin and Associate Prof. Dr. S. Ahmad for their assistance in collecting the clinical data, and the staff at the Universiti Kebangsaan Malaysia Medical Centre, especially N. F. Mujamil for her assistance in determining the fetal position through ultrasound scan.

\section{References}

Vaseghi, S.V. (2000). Advanced digital signal processing and noise reduction, Baffins Lane: John Wiley \& Sons Ltd

Widrow, B.; Glover Jr, J.R.; McCool, J.M.; Kaunitz, J.; Williams, C.S.; Hearn, R.H.; Zeidler, J.R.; Eugene, D. \& Goodlin, R.C. (1975). Adaptive noise cancelling: principles and applications, Proceedings of the IEEE, Vol. 63, pp. 1692-1716

Ifeachor, E.C. \& Jervis B.W. (2002). Digital signal processing: A practical approach, England: Prentice Hall 
Freeman, R.K.; Garite, T.J. \& Nageotte, M.P. (2003). Fetal heart rate monitoring, Lippincott William \& Wilkins

Philip, J.S. (2002). Fetal distress. Current Obstetrics \& Gynaecology, 12(1):5-21

Hershkovitz, R.; Sheiner, E. \& Mazor, M. (2002). Ultrasound in obstetrics: a review of safety, European Journal of Obtetrics \& Gynecology and Reproductive Biology, Vol. 101, pp. 1518

Karlsson, B.; Berson, M.; Helgason, T.; Geirsson, R.T. \& Pourcelot, L. (2000). Effects of fetal and maternal breathing on the ultrasonic Doppler signal due to fetal heart movement, European Journal of Ultrasound, pp. 47-52

Khandpur R. S. (2004). Biomedical Instrumentation: Technology and Applications, McGraw-Hill Professional

Najafabadi, F. S.; Zahedi, E. and Mohd Ali, M.A. (2006). Fetal heart rate monitoring based on independent component analysis, Computers in Biology and Medicine, Vol. 36, No. 3, pp. 241-252

Ramanujam, N.; Vishnoi, G.; Hielscher, A.H.; Rode, M.E.; Forouzan, I. \& Chance, B. (2000) Photon migration through the fetal head in utero using continuous wave, near infrared spectroscopy: clinical and experimental model studies, Journal of Biomedical Optics, pp. 163-172

Chance, B. (2005). Transabdominal Examination Monitoring and Imaging of Tissue. U.S. Patent 2005/0038344A1

Zourabian, A.; Chance, B.; Ramanujam, N.; Martha, R. \& David A.B. (2000). Transabdominal monitoring of fetal arterial blood oxygenation using pulse oximetry, Journal of Biomedical Optics, Vol. 5, pp. 391-405

Nioka, S.; Izzetoglu, M.; Mawn, T.; Nijland, M.J.; Boas, D.A. \& Chance, B. (2005). Fetal transabdominal pulse oximeter studies using a hypoxic sheep model, The Journal of Maternal-Fetal and Neonatal Medicine, Vol. 17, No. 6, pp. 393-399

Vintzileos, A.M.; Nioka, S. \& Lake, M. (2005). Transabdominal fetal pulse oximetry using near-infrared spectroscopy, American Journal of Obstetric \& Gynaecology, Vol. 192, pp. 129-133

Zahedi, E. \& Beng, G.K. (2008). Applicability of adaptive noise cancellation to fetal heart rate detection using photoplesthysmography. Computers in Biology and Medicine, Vol. 38, No. 1, pp. 31-41

Choe, R.; Durduran, T.; Yu, G.; Nijland, M.J.M.; Chance, B.; Yodh, A.G. \& Ramanujam, N. (2003). Transabdominal near infrared oximetry of hypoxic stress in fetal sheep brain in utero, Proceedings of the National Academy of Sciences, vol. 100, No. 22, pp. 12950-12954

Gan, K.B.; Zahedi, E. \& Mohd. Ali, M.A. (2009). Trans-abdominal fetal heart rate detection using NIR photopleythysmography: instrumentation and clinical results, IEEE Transactions on Biomedical Engineering, Vol. 56, No. 8, pp. 2075-2082.

Manolakis, D.G.; Ingle, V.K. \& Kogon, S.M. (2005). Statistical and adaptive signal processing. Norwood:Artech House, Inc.

Haykin, S. (2002). Adaptive filter theory. Prentice Hall.

Gan K.B. (2009). Non-invasive fetal heart rate detection using near infrared and adaptive filtering. Available online from: (http://ptsldigital.ukm.my) 
International Commission on Non-Ionizing Radiation Protection. (2000). ICNIRP statement on light-emitting diodes (LEDs) and laser diodes: Implications for hazard assessment, Health Phys., Vol. 78, No. 6, pp. 744-752

Bronzino, J.D. (2000). The biomedical engineering handbook: volume 1, Florida: CRC Press LLC

Allen, J. \& Murray, A. (2000). Variability of photoplethysmography peripheral pulse measurements at the ears, thumbs and toes, IEEE Proceeding Science and Technology, Vol. 147, No. 6, pp. 403-407 


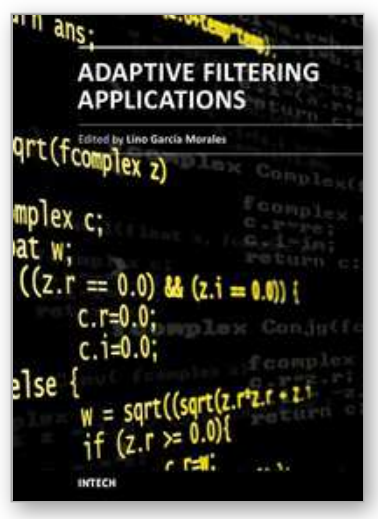

\author{
Adaptive Filtering Applications \\ Edited by Dr Lino Garcia
}

ISBN 978-953-307-306-4

Hard cover, 400 pages

Publisher InTech

Published online 24, June, 2011

Published in print edition June, 2011

Adaptive filtering is useful in any application where the signals or the modeled system vary over time. The configuration of the system and, in particular, the position where the adaptive processor is placed generate different areas or application fields such as: prediction, system identification and modeling, equalization, cancellation of interference, etc. which are very important in many disciplines such as control systems, communications, signal processing, acoustics, voice, sound and image, etc. The book consists of noise and echo cancellation, medical applications, communications systems and others hardly joined by their heterogeneity. Each application is a case study with rigor that shows weakness/strength of the method used, assesses its suitability and suggests new forms and areas of use. The problems are becoming increasingly complex and applications must be adapted to solve them. The adaptive filters have proven to be useful in these environments of multiple input/output, variant-time behaviors, and long and complex transfer functions effectively, but fundamentally they still have to evolve. This book is a demonstration of this and a small illustration of everything that is to come.

\title{
How to reference
}

In order to correctly reference this scholarly work, feel free to copy and paste the following:

Kok Beng Gan, Edmond Zahedi and Mohd. Alauddin Mohd. Ali (2011). Application of Adaptive Noise Cancellation in Transabdominal Fetal Heart Rate Detection Using Photoplethysmography, Adaptive Filtering Applications, Dr Lino Garcia (Ed.), ISBN: 978-953-307-306-4, InTech, Available from:

http://www.intechopen.com/books/adaptive-filtering-applications/application-of-adaptive-noise-cancellation-intransabdominal-fetal-heart-rate-detection-using-photop

\section{INTECH}

open science | open minds

\section{InTech Europe}

University Campus STeP Ri

Slavka Krautzeka 83/A

51000 Rijeka, Croatia

Phone: +385 (51) 770447

Fax: +385 (51) 686166

www.intechopen.com

\section{InTech China}

Unit 405, Office Block, Hotel Equatorial Shanghai

No.65, Yan An Road (West), Shanghai, 200040, China

中国上海市延安西路65号上海国际贵都大饭店办公楼 405 单元

Phone: +86-21-62489820

Fax: +86-21-62489821 
(C) 2011 The Author(s). Licensee IntechOpen. This chapter is distributed under the terms of the Creative Commons Attribution-NonCommercialShareAlike-3.0 License, which permits use, distribution and reproduction for non-commercial purposes, provided the original is properly cited and derivative works building on this content are distributed under the same license. 